Artigo Original

Original Article

Cristiane Moço Canhetti de Oliveira Gabriela Aparecida Fabbri Broglio² Ana Paula Lazarin Bernardes² Simone Aparecida Capellini ${ }^{3}$

Descritores

Fonoaudiologia

Fala

Diagnóstico

Distúrbios da fala Avaliação

Keywords

Speech, language and hearing sciences

Speech

Diagnosis

Speech disorders

Evaluation

Endereço para correspondência: Cristiane Moço Canhetti de Oliveira Av. Hygino Muzzi Filho, 737, Campus Universitário, Marília (SP), Brasil, CEP: 17525-900.

E-mail: cmcoliveira@marilia.unesp.br

Recebido em: 18/11/2011

Aceito em: 26/6/2012

\section{Relação entre taxa de elocução e descontinuidade da fala na taquifemia}

\section{Relationship between speech rate and speech disruption in cluttering}

Objetivo: Correlacionar a taxa de elocução com as rupturas da fluência em pessoas com taquifemia e comparar com pessoas sem taquifemia. Métodos: Participaram dessa investigação 14 indivíduos na faixa etária de 8 a 40 anos e 11 meses de idade, de ambos os gêneros, divididos em dois grupos pareados por idade e gênero. $\mathrm{O}$ GI foi composto por sete pessoas com taquifemia e o GII por sete pessoas sem taquifemia. Um protocolo de avaliação da fluência da fala foi utilizado para obter e analisar a amostra de fala, que considera a frequência das disfluências e a taxa de elocução. Resultados: Os dados indicaram que quanto maiores os fluxos de sílabas e de palavras por minuto, maior o número de rupturas na fala, tanto nas pessoas com taquifemia como nas pessoas sem taquifemia. Quanto à comparação entre os grupos, houve correlação tanto para sílabas por minuto como para palavras por minuto apenas no grupo de pessoas sem taquifemia. Conclusão: $\mathrm{O}$ grupo de taquifêmicos apresentou aumento na taxa de elocução e disfluências comuns excessivas. Nos dois grupos analisados ocorreu uma tendência em se obter maiores valores de disfluências comuns à medida que a taxa de elocução aumentava. Porém, na análise comparativa entre o grupo de pessoas com e sem taquifemia, a correlação foi significativa apenas no grupo de pessoas sem taquifemia.

\begin{abstract}
Purpose: To correlate speech rate and speech disruptions in individuals who clutter, and to compare with individuals who do not clutter. Methods: Participants were 14 individuals with ages between 8 and 40 years and 11 months, of both genders, divided into two groups paired by age and gender. GI was composed by seven individuals who clutter, and GII by seven individuals who do not clutter. The Speech Fluency Assessment Protocol was used to gather and analyze the speech samples, considering the frequency of speech disruptions and the speech rate. Results: Data indicated that the greater the rate of syllables and words per minute, the greater the number of speech disruptions, both for individuals who do and do not clutter. In the comparison between groups, there was correlation both for syllables and words per minute only in the group of individuals who not clutter. Conclusion: The individuals who clutter in this study presented a higher speech rate and frequency of common disfluencies. In both analyzed groups there was a tendency to greater frequency of common disfluencies as speech rate increased. However, in the comparative analysis between individuals who do and do not clutter, the correlation was significant only in the group of individuals who do not clutter.
\end{abstract}

Trabalho realizado no Laboratório de Estudos da Fluência, Departamento de Fonoaudiologia, Faculdade de Filosofia e Ciências, Universidade Estadual Paulista "Júlio de Mesquita Filho" - UNESP - Marília (SP), Brasil. Fonte de auxílio: PROEX-UNESP - Pró-Reitoria de Extensão, Universidade Estadual Paulista

(1) Departamento de Fonoaudiologia e Programa de Pós-Graduação em Fonoaudiologia, Faculdade de Filosofia e Ciências, Universidade Estadual Paulista "Júlio de Mesquita Filho" - UNESP - Marília (SP), Brasil.

(2) Departamento de Fonoaudiologia, Faculdade de Filosofia e Ciências, Universidade Estadual Paulista "Júlio de Mesquita Filho" - UNESP - Marília (SP), Brasil.

(3) Departamento de Fonoaudiologia e Programa de Pós-Graduação em Educação e em Fonoaudiologia, Faculdade de Filosofia e Ciências, Universidade Estadual Paulista "Júlio de Mesquita Filho" - UNESP - Marília (SP), Brasil.

Conflito de interesses: Não 


\section{INTRODUÇÃO}

Taquifemia e gagueira representam os principais distúrbios que apresentam rupturas na continuidade do fluxo da fala. Porém, enquanto gagueira é amplamente investigada, pesquisas que envolvem taquifemia são mais raras. Uma das possíveis justificativas para esse fato é a dificuldade na realização do diagnóstico desse distúrbio, bem como o fato da menor prevalência da taquifemia em relação à gagueira ${ }^{(1)}$.

Fluência é um aspecto de produção da fala que diz respeito à continuidade, suavidade, velocidade e/ou esforço, com as quais as unidades da linguagem fonológica, lexical, morfológica e/ou sintática são expressas ${ }^{(2)}$. Também foi descrita como um fenômeno multifacetado, composto por diversos elementos, como as disfluências, o esforço/tensão, a taxa de elocução e as pausas silenciosas ${ }^{(3)}$.

Portanto, taquifemia como um distúrbio da fluência, apresenta como principal manifestação disfluências excessivas na fala ${ }^{(4,5)}$. A taxa de elocução aumentada e/ou irregular é outro sintoma frequentemente associado ao quadro clínico do distúrbio ${ }^{(2,4,6-10)}$. Outra característica descrita pelos estudiosos é a inteligibilidade reduzida devido à coarticulação exagerada ou uma articulação imprecisa $^{(4,7)}$. Sabe-se também que o aspecto temporal da fala, ou a taxa de elocução, interfere na inteligibilidade da fala.

No quadro clínico da taquifemia ocorre a presença de disfluências comuns numa quantidade que chama a atenção do ouvinte $^{(2)}$. Um estudo que investigou as disfluências de dois adolescentes taquifêmicos mostrou que as disfluências comuns mais frequentes foram as interjeições e as revisões ${ }^{(11)}$. Essas disfluências parecem evidenciar dúvidas relacionadas à formulação linguística das frases ou pronúncia das palavras. Alguns estudiosos acreditam que estas disfluências ocorrem quando a pessoa com taquifemia fala muito rápido, disponibilizando tempo insuficiente para o falante organizar e formular as emissões ${ }^{(9)}$.

As manifestações da taquifemia são mais prováveis de serem percebidas durante a tarefa de fala mais espontânea, informal, menos estruturada, mais complexa linguisticamente, que envolve mais emoção e mais extensa ${ }^{(12)}$.

Portanto, taquifemia é um distúrbio complexo, com um amplo espectro de manifestações. Além das alterações na fala, a linguagem pode ser confusa ${ }^{(2,6-7)}$, o indivíduo taquifêmico frequentemente apresenta pouca ou nenhuma consciência do distúrbio ${ }^{(2,4)}$, e também pode apresentar manifestações na linguagem escrita, dificuldades na prosódia, entre outras características.

Portanto, a literatura ${ }^{(2,6,8-10)}$ apresenta informações sobre as manifestações da taquifemia, porém raros são os estudos que investigam a relação entre essas manifestações. Sendo assim, o presente trabalho tem por objetivo correlacionar a taxa de elocução com as rupturas da fluência em um grupo de pessoas com taquifemia e comparar com um grupo de pessoas sem taquifemia.

\section{MÉTODOS}

A presente pesquisa foi aprovada pelo Comitê de Ética em Pesquisa da Universidade Estadual Paulista (UNESP), Marília, sob o número 0529/2010. Todos os participantes (quando adultos) ou responsáveis (quando menores de 18 anos) assinaram o Termo de Consentimento Livre e Esclarecido antes de participar do estudo. Foram seguidas todas as recomendações da resolução 196/96 do Conselho Nacional de Saúde.

A amostra foi composta por 14 indivíduos na faixa etária de 8 anos a 40 anos e 11 meses de idade (média=34,5 anos), sendo oito do gênero feminino e seis do gênero masculino, divididos em dois grupos, com e sem taquifemia, pareados por idade e gênero.

O grupo de pessoas com taquifemia (GI) foi composto por sete indivíduos com taquifemia, sem qualquer outro déficit comunicativo, neurológico e cognitivo associado, que procuraram atendimento fonoaudiológico no Laboratório de Estudos da Fluência (LAEF) do Departamento de Fonoaudiologia da UNESP/Marília. Os critérios de inclusão de GI foram: (a) apresentar queixa de fala alterada, aumento da taxa de elocução, por parte dos pais nos casos infantis ou de adolescentes ou pelos próprios indivíduos (adultos); (b) manifestar fala disfluente, com excesso de disfluências comuns (acima de 10\%); (c) apresentar menos de 3\% de disfluências gagas, para ser descartada a possibilidade de ocorrer gagueira associada; (d) apresentar pouco ou nenhum concomitante físico associado à fala; (e) apresentar taxa de elocução maior do que os padrões esperados para a idade e gênero ${ }^{(13)}$, e; (f) escore acima de 120 no Inventário Preditivo de Taquifemia ${ }^{(12)}$, o que sugere diagnóstico de taquifemia.

O grupo de pessoas sem taquifemia (GII) foi composto por sete indivíduos fluentes, sem queixa de disfluência (atual e pregressa) por parte dos familiares, professores e/ou próprios indivíduos e sem qualquer déficit comunicativo, neurológico e cognitivo segundo as informações coletadas. Os critérios de inclusão dos participantes de GII foram: (a) apresentar menos de $10 \%$ de disfluências comuns e de $3 \%$ de disfluências gagas; (b) não apresentar antecedentes familiares para taquifemia e/ ou gagueira.

Uma amostra da fala de cada participante contendo no mínimo 200 sílabas fluentes foi obtida a partir de um estímulo visual de figura. O discurso dos indivíduos só pode ser interrompido (com perguntas e comentários), nos casos em que houve a necessidade de incentivar a produção, para que fosse alcançado o número necessário de sílabas para análise. Os casos foram filmados para análise e comparação dos achados, sendo utilizada uma filmadora digital Sony® e tripé. Após a coleta de fala dos participantes, as mesmas foram transcritas na íntegra, considerando-se as sílabas fluentes e não fluentes.

Para caracterizar a tipologia, a frequência das disfluências e a taxa de elocução foi utilizado o Protocolo de Avaliação da Fluência ${ }^{(14)}$, de acordo com a seguinte descrição:

- Disfluências comuns: hesitações, interjeições, revisões, palavras não terminadas, repetições de palavra, repetições de segmentos e repetições de frases;

- Disfluências gagas: repetições de sílabas, repetições de sons, prolongamentos, bloqueios, pausas e intrusões de sons ou segmentos.

A frequência das rupturas ou a porcentagem de descontinuidade de fala foi calculada a partir da contagem de todas as 
Tabela 1. Comparação intergrupos quanto à descontinuidade de fala, fluxo de sílabas e palavras por minuto

\begin{tabular}{|c|c|c|c|c|c|c|c|c|}
\hline Variável & Grupo & $\mathrm{n}$ & Média & DP & Mínimo & Máximo & Mediana & Valor de $p$ \\
\hline \multirow{2}{*}{ Descontinuidade de fala } & GI & 7 & 13,50 & 1,58 & 11,00 & 15,50 & 13,50 & \multirow{2}{*}{$0,002^{*}$} \\
\hline & GII & 7 & 6,36 & 0,80 & 5,00 & 7,50 & 6,50 & \\
\hline SPM & GII & 7 & 247,62 & 48,51 & 153,85 & 255,79 & 220,43 & $0,034^{*}$ \\
\hline PPM & GI & 7 & 179,43 & 26,30 & 150,00 & 257,14 & 175,70 & $0,001^{*}$ \\
\hline
\end{tabular}

*Valores significativos ( $\mathrm{p} \leq 0,05)$ - Teste de Mann-Whitney

Legenda: $\mathrm{GI}$ = grupo de taquifêmicos; GII = grupo sem taquifemia; DP = desvio-padrão; SPM = sílabas por minuto; $\mathrm{PPM}=$ palavras por minuto

disfluências. A taxa de elocução foi medida segundo o protocolo utilizado, caracterizando o fluxo de sílabas e de palavras por minuto $^{(14)}$.

Os indivíduos foram diagnosticados como taquifêmicos de acordo as manifestações consideradas obrigatórias do distúrbio pelos principais autores do assunto, que são taxa de elocução aumentada e disfluências comuns excessivas obtidas na avaliação da fluência ${ }^{(2,7,9,10)}$.

\section{Análise dos dados}

Os dados coletados foram submetidos à análise estatística, por meio da aplicação do Teste de Mann-Whitney, com o intuito de verificar possíveis diferenças entre os grupos estudados, para as variáveis de interesse. A correlação entre a taxa de elocução e a frequência de rupturas foi realizada por meio do Coeficiente de Spearman, que mede o grau de associação entre duas variáveis quantitativas de interesse. O coeficiente varia entre $-1 \mathrm{e}$ +1 . Quanto mais próximo destes dois extremos, maior será a associação linear entre as variáveis. Para este trabalho, o nível de significância adotado foi de $\mathrm{p}=0,05$.

\section{RESULTADOS}

Os resultados obtidos por meio da comparação das três medidas analisadas (porcentagem de descontinuidade de fala, fluxo de sílabas por minuto e fluxo de palavras por minuto) demonstraram diferença entre os grupos. O GI apresentou aproximadamente o dobro de disfluências, e um maior fluxo de sílabas e palavras por minuto em relação à GII (Tabela 1).

A Tabela 2 mostra a correlação entre a taxa de elocução (sílabas e palavras por minuto) e a descontinuidade na fala dos indivíduos de GI e GII. Nota-se na tabela que os coeficientes de correlação (r) foram positivos para ambos os grupos, mostrando que as duas variáveis contrapostas, a taxa de elocução e a descontinuidade de fala, apresentam comportamento paralelo, ou seja, quanto maior a taxa de elocução maior a frequência de rupturas. Portanto, tanto no grupo de pessoas com taquifemia quanto no de pessoas sem taquifemia parece existir uma correlação entre o aumento da taxa de elocução e o aumento das disfluências. No entanto, a correlação só foi significativa no grupo de pessoas sem taquifemia (GII).

Na Figura 1 verifica-se que quanto maior o fluxo de sílabas por minuto (SPM), ou também conhecido como taxa
Tabela 2. Correlações entre a descontinuidade de fala e a taxa de elocução (sílabas e palavras por minuto)

\begin{tabular}{lccc}
\hline \multirow{2}{*}{ Grupos Pares de variáveis } & $\begin{array}{c}\text { Coeficiente de } \\
\text { correlação }(r)\end{array}$ & Valor de $p$ \\
\hline \multirow{2}{*}{ GI } & Descontinuidade de fala X SPM & $+0,739$ & 0,058 \\
& Descontinuidade de fala X PPM & $+0,541$ & 0,210 \\
\hline \multirow{2}{*}{ GII } & Descontinuidade de fala X SPM & $+0,927$ & $0,003^{*}$ \\
& Descontinuidade de fala X PPM & $+0,782$ & $0,038^{*}$ \\
\hline
\end{tabular}

*Valores significativos $(p \leq 0,05)$ - Análise de Correlação de Spearman Legenda: $\mathrm{Gl}$ = grupo de taquifêmicos; $\mathrm{Gll}$ = grupo sem taquifemia; $\mathrm{SPM}$ = sílabas por minuto; PPM = palavras por minuto

de articulação, maior o número de rupturas na fala, tanto nas pessoas com taquifemia como nas pessoas sem taquifemia. Por exemplo, uma pessoa sem taquifemia com um fluxo de 243,8 sílabas por minuto (SPM), apresentou 7,5\% de disfluências, enquanto que outra pessoa sem taquifemia com um fluxo de 198,7 sílabas por minuto (SPM) apresentou 5\% de disfluências. Porém, a correlação foi significativa apenas para o grupo de pessoas sem taquifemia.

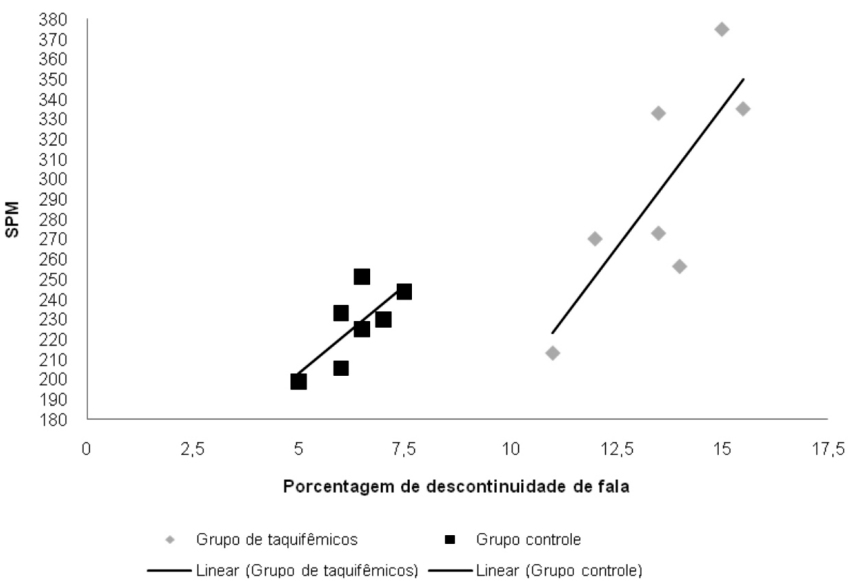

Figura 1. Correlação entre os valores de descontinuidade de fala e sílabas por minuto (SPM)

Em relação ao fluxo de palavras por minuto (PPM), que indica a taxa de informação produzida pelo falante, a análise estatística indicou que quanto mais palavras produzidas por minuto, mais disfluências ocorriam na fala de pessoas com taquifemia e de pessoas sem taquifemia (Figura 2). Por exemplo, 
um taquifêmico com um fluxo de 190,5 palavras por minuto (PPM), apresentou um total de 13,5\% de disfluências, enquanto que outro taquifêmico com um fluxo de 155,2 palavras por minuto (PPM) apresentou um total de $12 \%$ de disfluências. Porém, a correlação foi significante apenas para o grupo de pessoas sem taquifemia (GII).

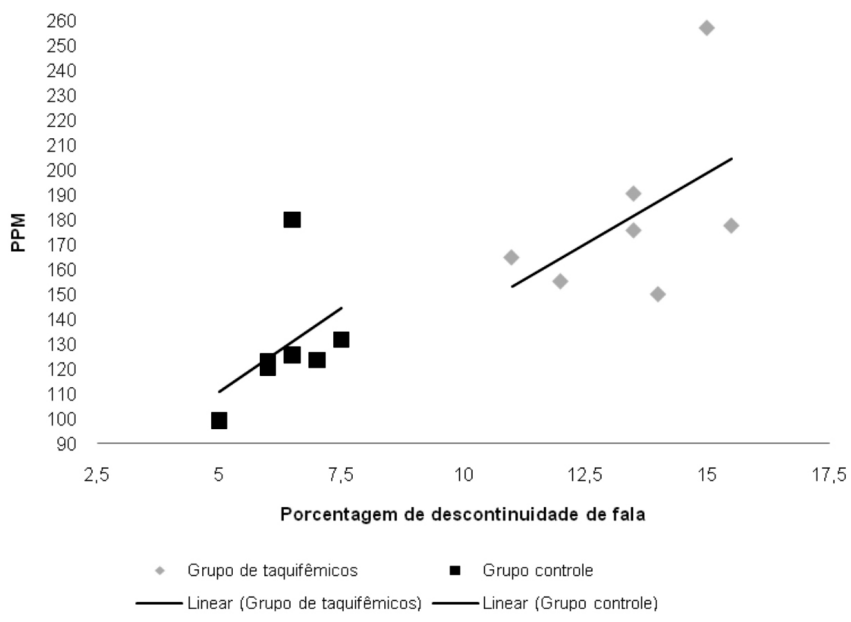

Figura 2. Correlação entre os valores de descontinuidade de fala e palavras por minuto (PPM)

\section{DISCUSSÃO}

O presente estudo analisou a correlação entre as principais manifestações do quadro clínico de taquifemia: disfluências e taxa de elocução aumentada. Para a realização desta análise, inicialmente foram medidas a frequência das rupturas e os fluxos de sílabas e de palavras por minuto. Conforme pesquisas anteriormente realizadas, taquifêmicos apresentaram maior ocorrência de rupturas em sua fala, caracterizadas principalmente por disfluências comuns ${ }^{(2,4,5,7,8,10)}$.

Os resultados encontrados neste trabalho concordam com os achados da literatura ${ }^{(2,4,8-10)}$, os quais revelam que taquifêmicos apresentam uma taxa de elocução aumentada. Tanto os fluxos de sílabas (SPM), como de palavras por minuto (PPM), apresentaram maiores no grupo de pessoas com taquifemia, em relação ao grupo de pessoas sem taquifemia. Em outras palavras, tanto a velocidade articulatória (SPM), como a produção de informação (PPM) foram aumentadas no grupo de pessoas com taquifemia.

A revisão bibliográfica destaca além da importância de medidas objetivas das disfluências e da taxa de elocução para a conclusão do diagnóstico de taquifemia, a necessidade de priorizar na terapia, a redução e o controle da taxa de elocução(7). Acredita-se que a diminuição da taxa de elocução propiciará a promoção da fluência, bem como a melhora na inteligibilidade da fala. Porém, a literatura também aponta uma lacuna sobre a correlação entre a taxa de elocução e a frequência de rupturas na fala de pessoas com taquifemia.

Neste sentido, o desenvolvimento deste trabalho permitiu afirmar que existe uma correlação positiva entre a taxa de elocução e a frequência de rupturas, tanto para pessoas com taquifemia como para pessoas sem taquifemia, indicando comportamento paralelo entre estas duas variáveis ${ }^{(15)}$. Este achado tem uma implicação clínica importante, uma vez que reforça a necessidade de priorizar na terapia de taquifêmicos a redução da taxa de elocução. Na literatura compilada não foram encontradas pesquisas para comparação destes $r$ esultados.

A diminuição da taxa de elocução favorece um aumento do tempo que o cérebro disponibiliza para processar a fala, o que propiciará ao falante taquifêmico mais tempo para organizar o pensamento, acessar o léxico, melhorar a precisão e amplitude articulatória, facilitando consequentemente à diminuição das disfluências comuns e o aumento na inteligibilidade da fala.

Outro apontamento importante refere-se ao fato da correlação ter sido significativa apenas no grupo de fluentes. No grupo de pessoas com taquifemia possivelmente outras variáveis também atuam em conjunto para que elas apresentem disfluências comuns excessivas. Pesquisadores destacaram que a taquifemia apresenta outras dimensões alteradas além da fala, como a linguagem, articulação, prosódia $^{(12)}$, que possivelmente estão inter-relacionadas e determinam a fluência ou disfluência na fala das pessoas com taquifemia.

Desta forma, os resultados desta investigação reforçam a necessidade de um olhar amplo para a taquifemia, direcionado às várias dimensões da comunicação, seja linguagem oral, linguagem escrita, habilidades auditivas desde o processo diagnóstico, propiciando assim uma terapia mais eficaz.

Acreditamos que este estudo é de extrema relevância na área dos distúrbios da fluência, uma vez que é escassa a literatura específica sobre essa temática, sinalizando que os achados aqui descritos podem contribuir para o conhecimento científico da Fonoaudiologia e áreas afins.

\section{CONCLUSÃO}

Pessoas com taquifemia apresentaram aumento na taxa de elocução e disfluências comuns excessivas. Além disso, houve tendência em se obter maiores valores de disfluências comuns à medida que a taxa de elocução aumentava. Porém, na análise comparativa entre os grupos de pessoas com e sem taquifemia, essa correlação foi significativa apenas no grupo de pessoas sem taquifemia.

Vale ressaltar que determinar os valores objetivos da frequência de rupturas e da taxa de elocução, bem como compreender a correlação existente entre essas variáveis são aspectos importantes do diagnóstico da taquifemia. A avaliação da linguagem, da articulação e da prosódia também é fundamental para auxiliar no diagnóstico e no planejamento terapêutico de pessoas com taquifemia, tendo em vista a complexidade do distúrbio.

\footnotetext{
* CMCO foi responsável pela coleta, tabulação dos dados e elaboração do artigo; GAFB foi responsável pela coleta, análise da amostra de fala e tabulação dos dados; APLB foi responsável pela coleta e análise da amostra de fala; SAC contribuiu com a conclusão dos achados e elaboração do artigo.
} 


\section{REFERÊNCIAS}

1. Daly DA, Burnett ML. Cluttering: another fluency syndrome. In: Curlee RF. Stuttering and related disorders of fluency. New York: Thieme Medical Pub; 1993. p. 179-204.

2. ASHA. Special Interest Division 4: Fluency and Fluency Disorders. Terminology pertaining to fluency and fluency disorders: Guidelines. ASHA Suppl. 1999 Mar-Apr;41(2 Suppl 19):29-36.

3. Oliveira AMCC, Ribeiro IM, Merlo S, Chiappetta ALML. O que fonoaudiólogos e estudantes de fonoaudiologia entendem por fluência e disfluência. Rev CEFAC. 2007 Mar;9(1):40-6.

4. St Louis KO, Raphael LJ, Myers FL, Bakker K. Cluttering updated. The ASHA Leader, ASHA. 2003;4,20-2.

5. Myers FL, Bakker K, St Louis KO, Raphael LJ. Disfluencies in cluttered speech. J Fluency Disord. 2012 Mar;37(1):9-19.

6. Daly DA, Burnett ML. Cluttering: assessment, treatment planning, and case study illustration. J Fluency Disorder. 1996 SepDec;21(3-4):239-48.

7. St. Louis KO, Myers FL. Clinical management of cluttering. Lang Speech Hear Serv Sch. 1995 Apr;26:187-95.
8. St. Louis KO. Global perspectives on cluttering: research, assessment and treatment. Paper presented at: $6^{\text {th }}$ World Congress on Fluency Disorders, Rio de Janeiro, Brasil. 2009.

9. Van Zaalen Y, Wijnen F, De Jonckere PH. Differential diagnostic characteristics between cluttering and stuttering - part one. J Fluency Disord. 2009 Set;34(3):137-54.

10. Oliveira CMC, Bernardes APL, Broglio GAF, Capellini SA. Perfil da fluência de indivíduos com taquifemia. Pró-Fono R Atual Cient. 2010 Dec;22(4):445-50.

11. Myers FL, St Louis KO. Two youths who clutter, but is that the only similarity? J Fluency Disord. 1996 Sep-Dec; 21(3-4):297-304.

12. International Cluttering Association [Internet]. Pennsylvania: International Cluttering Association- ICA, [atualizado 2009 Set 19; citado 2009 Dez 14]. Disponível em: http://associations.missouristate. edu/ICA/

13. Andrade CRF, Martins VO. Perfil evolutivo da fluência da fala de falantes do português brasileiro. Pro Fono. 2008 Jan-Mar;20(1):7-12.

14. Andrade CRF. Protocolo para avaliação da fluência da fala. Pro Fono. 2000;12(2):131-4.

15. Zou KH, Tuncall K, Silverman SG. Correlation and simple linear regression. Radiology. 2003;227(3):617-22. 